\title{
Ruhestandsmigration und Reurbanisierung
}

\author{
Trends in Deutschland 1995-2012
}

\author{
Uwe Engfer ${ }^{1}$ (iD)
}

Eingegangen: 7. Juni 2017 / Angenommen: 26. Oktober 2017 / Online publiziert: 2. November 2017

(C) Springer-Verlag GmbH Deutschland 2017

Zusammenfassung In der Diskussion über die aktuellen Tendenzen der Binnenmigration der älteren Generation gibt es eine Kontroverse über einen möglichen Trendwechsel. Für frühere Generationen von Personen im Ruhestand ist in vielen Untersuchungen (in verschiedenen Ländern) nachgewiesen worden, dass ihre Umzüge eine Art counterurbanisation waren. Im Saldo zog die ältere Bevölkerung aus den Kernen der Agglomerationen an die Peripherie oder generell aus den Städten in die ländlichen Regionen. Strittig ist nun, ob es dieses Muster der Ruhestandsmigration weiterhin gibt, oder ob es neuerdings auch die Älteren zurïck in die Städte zieht. Obwohl dieser neue Trend in den Medien, in der Politik und in der Immobilienwirtschaft häufig als Tatsache hingestellt wird, gibt es wenig empirische Belege dafür. In der hier vorgestellten Untersuchung wird der Frage nach dem Trendwechsel zur Reurbanisierung mit einer Aggregatdaten-Analyse nachgegangen. Basis ist eine Auswertung der Indikatoren und Karten zur Raum- und Stadtentwicklung (INKAR) für die Periode 1995-2012. Ergebnis der Analysen ist, dass es in Deutschland in der Tat einen bemerkenswerten Strukturwandel der Ruhestandsmigration gibt. Die ,Stadtflucht" der Senioren ist deutlich zurückgegangen und es gibt immer mehr Großstädte (insbesondere in Ostdeutschland), die für diese Bevölkerungsgruppe positive Migrationssalden aufweisen.

Schlüsselwörter Ruhestandsmigration · Reurbanisierung · Wohnortwechsel · Altenwanderung · Sozialgeographie des Alterns

Dr. Uwe Engfer

engfer@ifs.tu-darmstadt.de

1 Institut für Soziologie, Technische Universität Darmstadt, Dolivostraße 15, 64293 Darmstadt, Deutschland

\section{Retirement Migration and Reurbanisation}

Trends in Germany 1995-2012

Abstract In the debate about recent trends in elderly migration in Germany a controversy arose about a possible change of patterns. With respect to former generations of retired persons many studies (in different countries) gave evidence of the fact that a change of residence in retirement in many instances was a 'counterurbanisation'. On balance the elderly population left the central cities of agglomerations and went to the peripheral regions or more generally from urban to rural regions. At issue is now whether this pattern of retirement migration still exists or whether seniors nowadays move into the cities as well. In the media, in politics and in real estate business the suspected trend is often treated as a matter of fact, but empirical evidence is still rare. The study presented here investigates the suspected change towards reurbanisation in retirement migration with an analysis of aggregate data. The data base INKAR (indicators and maps of spacial and urban development) is used to examine developments from 1995 to 2012. The results show that there is indeed a structural transformation of elderly migration in Germany. The urban outmigration of seniors has decreased considerably and more and more big cities (especially in East Germany) turn up with a surplus of in-migration of older adults.

Keywords Retirement migration - Reurbanisation · Changing residence $\cdot$ Elderly migration $\cdot$ Geography of aging 


\section{Umzüge im Ruhestand als Forschungsthema}

Für einen Großteil der Bevölkerung in den entwickelten Ländern hat sich in der zweiten Hälfte des 20. Jahrhunderts die ,Normalbiographie“ positiv verändert: Zwischen dem Ende der Erwerbsarbeit und dem Tod ist ein neuer Lebensabschnitt entstanden, den man vereinfacht als „Ruhestand“ bezeichnen kann. Infolge weiter steigender Lebenserwartung dehnt sich diese Phase immer weiter aus und die Lebensqualität im Alter ist über mehrere Jahrzehnte angestiegen. Im Vergleich zu früheren Generationen sind Menschen im Ruhestand deutlich gesünder, sie sind ökonomisch vergleichsweise gut abgesichert und sie sind besser gebildet. Für die meisten verdient der „Ruhestand“ auch seinen Namen, denn erwerbstätig sind in diesem Lebensabschnitt nur die Wenigsten. Die Arbeit beschränkt sich auf Haushaltsund Familienarbeit (Statistisches Bundesamt 2015: 31 ff.).

Vor diesem Hintergrund wird diese Lebensphase häufig als eine Zeit der „späten Freiheit“ (Rosenmayr 1983) bezeichnet. Die Menschen sind - anders als in früheren Lebensabschnitten - freier bei der Wahl der Aktivitäten, mit denen sie ihre Zeit verbringen. Und sie sind freier in der Wahl des Ortes, an dem sie leben möchten, denn die Bindung an einen bestimmten Arbeitsplatz und Betrieb fällt weg. Wie diese letztgenannte Freiheit zur räumlichen Mobilität genutzt wird, ist Thema eines speziellen Zweiges der Migrationsforschung: der Ruhestandsmigrationsforschung.

Migration im späteren Erwachsenenalter ist zwar seit jeher ein bekanntes Phänomen in Industriegesellschaften, aber vor 1970 ist diesem Thema kaum wissenschaftliche Aufmerksamkeit gewidmet worden, weil nur ein sehr kleiner Teil der älteren Bevölkerung tatsächlich umgezogen ist und die Distanz dieser Umzüge meist kurz war. Dabei geht es an dieser Stelle natürlich nur um die freiwilligen Umzüge. Flucht, Vertreibung und andere Formen erzwungener Migration werden hier ausgeklammert.

Zwischen den 1970er- und 1990er-Jahren ist in Nordamerika, speziell in den USA, das Phänomen allerdings deutlich sichtbar geworden: Die Zahl der Umzüge im späteren Erwachsenenalter nahm stark zu. Die Umzüge führten über zum Teil große Distanzen und im Gefolge dieser neuartigen Binnenmigration entstanden teilweise hochgradig alterssegregierte Siedlungsformen (,Rentnerstädte'). Im Zentrum der Aufmerksamkeit standen die neuartigen Bevölkerungsverschiebungen in der Altersgruppe der Senioren weg von den industrialisierten, großstädtischen Metropolregionen des Nordens und Nordostens der USA in die sonnenreichen Staaten des Südens, z. B. Florida und Arizona (vgl. als Überblick Longino 1995). Diese auf der Basis von hochaggregierten Zensusdaten ermittelten Migrationsströme ließen den Eindruck entstehen, dass die Ruhestandsmigration durch mehrere Merkmale charakterisiert sei: Sie führt über große Distanzen und die Akteure sind jünge- re Personen der älteren Generation, die einen als negativ empfundenen Herkunftswohnsitz freiwillig verlassen, um in einer Zielregion, die mehr Annehmlichkeiten bietet, eine lange Zeit des Ruhestands zu verleben. Sehr schnell hat die Forschung sich dann aber bemüht, dieses Zerrbild der Wohnstandortverlagerungen im Alter zu korrigieren.

Litwak und Longino (1987) führten in diesem Zusammenhang die Unterscheidung von drei typischen Umzugsarten im Alter ein, die jeweils charakteristisch für eine bestimmte Phase des Alters seien. Der erste Typ sind Umzüge der ,jungen Alten“, die meist kurz nach dem Übergang in den Ruhestand stattfinden. Umgezogen wird gemeinsam mit der Partnerin oder dem Partner. Die Zielregionen werden ausgewählt, weil man sich dort eine bessere Lebensqualität verspricht: eine gesündere Umgebung, bessere Möglichkeiten für Freizeitaktivitäten und einen Lebensstil nach dem Modell „Urlaub für immer“. Der zweite Typ von Umzug im Alter findet tendenziell im etwas späteren Seniorenalter statt. Er wird ausgelöst durch nachlassende Gesundheit und steigenden Hilfebedarf. Vom Umzug verspricht man sich bessere Unterstützung durch Familienmitglieder oder andere soziale Netzwerke. Auslöser sind oft kritische Lebensereignisse wie der Tod des Partners oder der Ausbruch einer schweren Krankheit. Der dritte Typ von Umzug im Alter ist dann der mehr oder weniger erzwungene Umzug zu einem Zeitpunkt, an dem man nicht mehr in der Lage ist, einen unabhängigen Haushalt zu führen. Diese unfreiwilligen Umzüge führen meist in Gemeinschaftsunterkünfte oder andere betreute Wohnformen. Andere Autoren haben in ähnlicher Form die Theorie der Ruhestandsmigration differenziert (Wiseman 1980; Serow 1987) und gezeigt, dass es sehr heterogene Bedingungs- und Ursachenfaktoren sind, die hinter dieser Form der Binnenmigration stehen. Die Push- und Pull-Faktoren der Ruhestandsmigration unterscheiden sich grundsätzlich von den Faktoren, die in früheren Phasen des Lebenslaufes wirksam sind. Das positive Bild der annehmlichkeitsorientierten Wanderer (amenity migrants) ist nur eine Seite der Medaille. Auf der anderen Seite gibt es auch die mehr oder weniger unfreiwilligen Umzüge, die durch den Rückgang der Einkommen nach Ende der Erwerbsarbeit, zu hohe Mieten oder durch ungesunde Umweltbedingungen in heruntergekommenen Wohnquartieren mit Lärm, Luftverschmutzung und hoher Kriminalität oder durch das Angewiesensein auf die Hilfe anderer erzwungen werden.

Neben der Frage nach Motiven und Anlässen der Ruhestandsmigration wurde von Beginn an auch die Frage der räumlichen und demographischen Folgen dieses neuen Migrationstyps untersucht (vgl. Longino 1980; Golant 1987; Longino 1995). Dabei wurde klar, dass die Vorstellung, es seien vor allem Fernumzüge, und die Vorstellung, das Phänomen werde quantitativ immer bedeutsamer, falsch sind. Die meisten Umzüge führen nur über kurze Distanzen, und 
es wird herausgestellt, dass die Älteren im Vergleich zu anderen Altersgruppen die sesshafteste Gruppe sind (Golant 1987). Friedrich stellt auf der Basis seiner Analysen fest, dass beide Punkte in besonderem Maße für Deutschland gelten (Friedrich 1994; Friedrich 1995). Darüber hinaus zeigen seine Daten (aus 1991/1992), dass in Deutschland die mobilste Gruppe nicht die jungen, sondern die alten Senioren sind. „Damit widersprechen bislang alle Befunde hinsichtlich der Altersbeteiligung, der Herkunfts- und Zielpräferenzen, der Reichweiten sowie der Austauschmuster der verbreiteten Einschätzung, wonach Wohnsitzwechsel im Alter in der Regel Fernwanderungen zu attraktiven Ruhesitzregionen seien und vorrangig von jüngeren Senioren unternommen würden“ (Friedrich 1994: 414).

Im Hinblick auf die Frage nach der Lebensqualität im Alter ist die Ruhestandsmigration also ein höchst ambivalentes Phänomen. Ein steigendes Niveau dieser Art von Binnenmigration kann - auf der einen Seite - sowohl Indikator sein für einen Anstieg der Chancen und Ressourcen, im Ruhestand einen frei gewählten Platz zu finden, an dem man einen Lebensstil ganz nach eigenem Geschmack praktizieren kann. Die Zunahme der Ruhestandsmigration kann jedoch - auf der anderen Seite - auch bedeuten, dass es einer Gesellschaft - zumindest in bestimmten Regionen nicht gelingt, Lebensumstände zu gewährleisten, die es älteren Menschen erlauben, am angestammten Platz in guter Lebensqualität alt zu werden. Fragt man nach den räumlichen Charakteristika der Quell- und Zielgebiete der Ruhestandsmigration, gibt es durchgängig einen auffallenden Befund. Er lautet in der Formulierung von Friedrich (1994: 413): die „Tendenz zur Dekonzentration entgegen der metropolitanen Hierarchie“ oder bei Golant (1987: 534): ,nonmetropolitan areas have experienced net migration gains of elderly people“. Stets ziehen (im Saldo) die Älteren aus den Kernstädten ins Umland oder - bei Fernumzügen in ländliche Regionen oder in das suburbane Umland entfernter Metropolregionen. Man könnte auf die Idee kommen, das Ausmaß der Ruhestandsmigration und insbesondere das negative Saldo bei der Altenwanderung als Messgröße für die (Un-)Attraktivität einer Stadt zu verwenden. Umgekehrt könnte man die Abschwächung oder das Verschwinden dieser Art von „Stadtflucht“ als Indikator für eine zunehmende Attraktivität der Städte auch für die ältere Generation werten. In ihrer Übersicht zur nordamerikanischen Ruhestandsmigrationsforschung verweisen Northcott und Petruik (2011: $313 \mathrm{f}$.) darauf, dass - immer noch die allermeisten Senioren nicht umziehen und dass es begründete Vermutungen gibt, dass die gegenwärtig alternde Generation an ihren zunehmend „Senior-friendly“-Wohnstandorten bessere Bedingungen für das „aging in place“ vorfindet als frühere Generationen.

Vor diesem Hintergrund soll im vorliegenden Beitrag der Frage nachgegangen werden, wie sich die Salden der Ru- hestandsmigration in Deutschland in der Periode 1995 bis 2012 verändert haben. Die Analyse soll den Nachweis erbringen, dass sich ein Strukturwandel der Ruhestandsmigration weg von der ,Dekonzentration entgegen der metropolitanen Hierarchie“ (Friedrich 1994: 413) abzeichnet und dass dieser Strukturwandel als eine Facette des Prozesses der Reurbanisierung verstanden werden kann. In Kapitel 2 stelle ich die Befunde der Ruhestandsmigrationsforschung in den Kontext des Counterurbanisierungs- und Reurbanisierungsdiskurses. In Kapitel 3 werden der methodische Ansatz, die Datengrundlage und erste zentrale Ergebnisse vorgestellt. In Kapitel 4 werden die Veränderungen der Ruhestandsmigrationssalden in kreisfreien Großstädten Deutschlands beschrieben und auf der Basis eines multivariaten Regressionsmodelles wird exploriert, welche Faktoren zu positiven oder negativen Salden führen. In Kapitel 5 werden die Befunde diskutiert und interpretiert.

\section{Counterurbanisation oder Reurbanisierung?}

Ein zentrales Element der Entstehung und Entwicklung moderner Industriegesellschaften war die Urbanisierung, das heißt die siedlungsstrukturelle Konzentration der Bevölkerungen in (immer größer werdenden) Städten. Erst in den 1970er-Jahren gerät in den USA in den Blick, dass es auch gegenläufige Entwicklungen gibt: Dekonzentrationstendenzen. Berry (1976) prägte für diese neue Art siedlungsstrukturellen Wandels den Begriff „counterurbanization“. Der Begriff wurde schnell aufgegriffen, und es entwickelte sich eine Jahrzehnte andauernde und international vergleichende Forschungstradition (vgl. als Übersicht Mitchell 2004). Mit dem Begriff bezeichnet wurden allerdings sehr heterogene empirische Tatbestände. Teils wurden Abwanderungen von Kernstädten ins Umland oder in ländliche Regionen betrachtet, teils wurden relative Wachstumsraten unterschiedlich konzentrierter Regionen verglichen, teils wurden Bevölkerungsbestände zu verschiedenen Zeitpunkten oder in verschiedenen geographischen Regionen verglichen. Mitchell (2004) hat den überzeugenden Vorschlag gemacht, eindeutiger zwischen drei verschiedenen Begriffsverwendungen zu unterscheiden. Erstens bezeichnet der Begriff einen strukturellen Prozess, in dem sich - durch komplexe demographische Veränderungen - eine weniger konzentrierte Siedlungsweise entwickelt. Zweitens meint counterurbanisation ein resultierendes Struktur-Muster der Verteilung von Siedlungsformen auf einem Territorium, das heißt den Übergang zu einem weniger konzentrierten Muster zu einem späteren Zeitpunkt. Und drittens ist mit dem Begriff eine konkrete Migrationsbewegung gemeint, das heißt der Nachweis, dass eine bestimmte Bevölkerungsgruppe im Saldo in dekonzentriertere Siedlungsformen abwandert. Wenn es um counterurbanisation dieses Typs geht, dann 
sind - nach Mitchell (2004: 24) - drei Gruppen als Akteure identifiziert worden: zum einen Normalbürger, die in den städtischen Zentren aus den unterschiedlichsten Gründen weder arbeiten noch wohnen wollen, zum zweiten Anhänger einer Alternativkultur, die nach dem Motto „Zurück zur Natur/aufs Land" leben wollen, und schließlich zum dritten die Ruhestandsmigranten.

Ob es counterurbanisation in den erwähnten verschiedenen Ausprägungen nicht nur in den USA, sondern auch in den vergleichbar entwickelten europäischen Ländern gibt, war lange umstritten (vgl. Kontuly/Wiard/Vogelsang 1986: 170). Mit der Zunahme des Kenntnisstandes zur Ruhestandswanderung wurde aber immer deutlicher, dass diese Form der counterurbanisation auch in vielen europäischen Ländern vorzufinden ist. In England und Schottland gibt es diese Abwanderung aus den Städten an die Küsten und in ländliche Regionen ebenso wie in den Niederlanden und Frankreich (vgl. Cribier 1980; Warnes/Law 1983; Lewis/ McDermott/Sherwood 1991; Rees 1992; Fokkema 1996; Stockdale/Findlay/Short 2000). Die Studie von Cribier und Kych (1993) zum Vergleich der Ruhestandsmigration aus London und Paris nimmt den Fortzug aus Städten sogar zum Definitionsmerkmal dieser Art von Migration. ${ }^{1}$ Bonaguidi und Abrami (1992) haben Turin und Mailand untersucht und schlussfolgern: „Although the non-elderly represent the dominant component of the deconcentration process within metropolitan areas, the elderly are important contributors to the net shift of population from metropolitan areas. There is some evidence that this metropolitan age-selective shift has been intensifying in more recent years" (Bonaguidi/Abrami 1992: 161). Die Größe der negativen Salden bei der Ruhestandsmigration variiert zwar stark zwischen den Stadtregionen und die Push- und Pull-Faktoren sind sehr unterschiedlich, aber das Resultat war in allen Ländern ähnlich: Die Migration der älteren Generation trägt zur Dekonzentration der Bevölkerung bei.

In der Bundesrepublik gab es nur wenige Studien, in denen die Ruhestandswanderung im Zentrum stand (vgl. als Übersicht Rohr-Zänker 1989). Die internationale Migration unter den Senioren (etwa Umzüge an die Mittelmeerküsten oder in die touristisch attraktiven Regionen der Alpen) war quantitativ sehr gering, und - anders als in den USA - war die Ruhestandsmigration auch nicht mit der Entste-

\footnotetext{
1 „We define a retirement migration as a move away from the urban area following the end of working life. Retirement migration does not therefore, include all migration in the retired population " (Cribier/ Kych 1993:1404).
}

hung völlig neuer Siedlungstypen verbunden. ${ }^{2}$ Auf der Suche nach counterurbanisation-Tendenzen wurde man aber auch hier fündig, wenn man die Migrationsstatistiken nach Altersgruppen disaggregierte: „A disjunct urbanization pattern for the younger group, a counterurbanization pattern for the older group resulted" (Kontuly/Wiard/Vogelsang 1986: 179). Und trotz des relativ geringen Volumens der Ruhestandsmigration in einigen Regionen Deutschlands war der Einfluss auf die Bevölkerungsentwicklung messbar: „The in-migration of the older age-group influences the growth of peripheral regions around the North and Baltic Seas, Lüneburg, parts of the Black Forest, the Alps regions of the South, the Alpine Foreland, and the Bavarian Forest" (Kontuly/Wiard/Vogelsang 1986: 179). Walther (2007: 277) fasst den Erkenntnisstand folgendermaßen zusammen: „Auch die meisten Alterswanderungen weisen von den Kernstädten weg: die Fernwanderungen in die Regionen mit Klima- und Landschaftsgunst (,Ruhestandsregionen'). Es setzt eine allmähliche Dekonzentration der Alterung ein: die Alterung ,geht in die Fläche“".

Auch in einer neueren Übersicht von Friedrich (2008) zu den Binnenwanderungen älterer Menschen, in der die Verflechtungsmatrizen von Quell- und Zielgebieten analysiert werden konnten, wird einerseits die Stabilität der alten Muster betont, es wird aber auch darauf hingewiesen, dass es Indizien für graduelle Veränderungen gibt. „Das noch Mitte der 1990er-Jahre nahezu durchgängige Bild, dass vor allem die größeren Kernstädte zugunsten der angrenzenden Gebietseinheiten innerhalb der Verdichtungsräume zu negativen Wanderungssalden tendierten, hat seine Gültigkeit eingebüßt: Auch im suburbanen Umland des Ruhrgebiets, des Rhein-Main-Gebiets oder von Stuttgart verringert sich die Seniorenbevölkerung durch Fortzüge, und keineswegs sind immer noch die meisten mittleren Kernstädte durch Binnenwanderungsverluste gekennzeichnet" (Friedrich 2008: 190). Friedrich will mit diesem Hinweis auf graduelle Veränderungen im Bereich der Ruhestandsmigration vor allem Erwartungen dämpfen, dass ländliche Regionen durch zuwandernde Senioren wirtschaftlich und demographisch profitieren könnten.

Die genauere Analyse dieser sich andeutenden Veränderungen im Bereich der Ruhestandsmigration ist das Ziel des vorliegenden Beitrags. Geprüft werden soll eine Hypothese, die im Verlauf des seit einigen Jahren laufenden Diskurses um die neuerliche Bedeutungszunahme der Städte, die „Reurbanisierung“, geäußert worden ist. Angesichts der

\footnotetext{
2 Städte nach dem Vorbild der Del-Webb-Städte (Del E. Webb, geb. 1899, Bauunternehmer, erfand die Seniorenstadt mit Lifestyle wie z. B. Sun City in der Phoenix-Metropolregion; vgl. http://www.delwebb. com (20.09.2017)) sind in Deutschland schon aus rechtlichen Gründen nicht zulässig, ganz abgesehen davon, dass sie wegen des immensen Flächenverbrauchs auch aus ökonomischen Gründen als nicht realisierbar erscheinen (vgl. Eizenhöfer/Link 2006).
} 
Tatsache, dass in der Vergangenheit die Ruhestandsmigration stets als Beispiel für Counterurbanisation-Tendenzen galt, wird neuerdings überraschenderweise behauptet, dass es die ältere Generation in die Städte ziehe. Glasze und Graze (2007: 467) zitieren einschlägige Behauptungen aus den Medien. ${ }^{3}$ Danach seien die wohlhabenden Suburbaniten zum Verkauf ihrer nach dem Aus- und Wegzug der Kinder überdimensionierten Eigenheime im eintönigen Suburbia bereit, um zentraler in der Stadt zu wohnen und dort die nahegelegenen Annehmlichkeiten der Gastronomie, der kulturellen Einrichtungen und der medizinischen Versorgung zu nutzen. Ähnliche Behauptungen gibt es aus der Stadtentwicklung und Immobilienwirtschaft: „Neben den jungen Leuten zieht es aber verstärkt auch Ältere wieder in die Städte. [...] Es ist in den letzten Jahren deutlich zu beobachten, dass diese ,jungen" Alten vermehrt altersgerechtes Wohnen in der Innenstadt suchen, um nah am gesellschaftlichen Leben zu sein und gleichzeitig besser von der Infrastruktur in der Stadt (Einkaufsmöglichkeiten, Ärzteversorgung, Kulturangebote, ÖPNV etc.) zu profitieren“ (Spars 2012: 50). Auch Autoren, die auf soziologische Befunde zum Wert- und Lebensstilwandel der künftigen Seniorengeneration Bezug nehmen, postulieren einen Wandel in diese Richtung (Opaschowski 2005; Poddig 2006).

Dass es in Deutschland, wenn man die Bevölkerung insgesamt betrachtet, deutliche Tendenzen der Reurbanisierung, einer „Renaissance der Städte“, gibt (zumindest in der quantitativ-demographischen Dimension), kann inzwischen als nachgewiesen gelten (vgl. Brühl/Echter/Frölich von Bodelschwingh et al. 2005; Brake/Herfert 2012; Osterhage/Kaup 2012; Milbert 2017). Die lange Zeit nur, gefühlte " Trendumkehr weg von der Suburbanisierung manifestiert sich dabei in unterschiedlicher Form, je nachdem, ob es sich um wachsende, stagnierende oder schrumpfende Städte handelt (Herfert/Osterhage 2012). Unklar ist jedoch, ob auch die ältere Generation aktiv zum Reurbanisierungstrend beiträgt. Die entsprechenden Behauptungen sind bislang nicht ausreichend empirisch belegt. Teils wird die Altenwanderung nur am Rande behandelt, weil sie das Volumen der Gesamtwanderung nur gering beeinflusst (Milbert 2017), teils wird die hochgradige Altersselektivität der Migrationsströme als besonderes Kennzeichen der Reurbanisierung herausgestellt. Der bei der Analyse der Reurbanisierungstendenzen in Ostdeutschland ermittelte Befund von Köppen, Mai und Schlömer (2007: 222) ist vermutlich auch für Westdeutschland nicht ganz unzutreffend: „Strom und Gegenstrom werden von verschiedenen Altersgruppen do-

\footnotetext{
${ }^{3}$ Ihre eigene Untersuchung, eine Befragung von Mainzer Suburbaniten, kommt zu dem Ergebnis, dass es zwar ein gewisses Potenzial für diese Art von Umzügen gibt, aber die große Mehrheit der Befragten doch im Umland wohnen bleiben möchte (Glasze/Graze 2007: 472).
}

miniert: Junge Erwachsene ziehen tendentiell in die Städte, Familien und Ältere aus den Städten heraus."4

Der Begriff Reurbanisierung bezeichnet im vorliegenden Beitrag ein rein quantitativ-demographisches Phänomen: die absolute oder relative Bedeutungszunahme der Städte durch Veränderungen des Bevölkerungsstandes. Dabei interessieren hier nur die Migrationsströme der Bevölkerung im Alter von 65 Jahren und älter. Für diese Altersgruppe wird analysiert, wie sich die Salden der Zuzüge und Fortzüge (bezogen auf 1.000 Einwohner) in bzw. aus kreisfreien Großstädten in Deutschland entwickelt haben. Dabei verwende ich einen sehr weiten Reurbanisierungsbegriff. Nicht nur Zuwanderungsgewinne durch die Ruhestandsmigration sind für mich ein Indikator, dass die Älteren zur Reurbanisierung beitragen, sondern auch schon das Verschwinden der traditionellen Negativsalden. Dieser Indikator „,Saldoraten der Zu- und Fortzüge der Älteren Ruhestandswanderer $65+$ " ist in zweifacher Hinsicht unbefriedigend, um die Befunde sachgerecht tiefergehend interpretieren $\mathrm{zu}$ können. Zum einen lässt er keine differenzierte Betrachtung zu, um welche der oben erwähnten Typen von Ruhestandsmigration es sich handelt (z. B. junge oder alte Senioren), zum anderen gibt er keine Auskunft darüber, ob es sich um Umzüge im Nahbereich oder um Fernumzüge handelt. Ebenso nicht möglich sind auf der Basis dieses Indikators Aussagen über Veränderungen des Volumens der Ruhestandsmigration und Aussagen über die Verflechtung der Quell- und Zielgebiete der Wanderungen. Trotz dieser Einschränkungen, die allein der begrenzten Datenverfügbarkeit der verwendeten Datenbasis geschuldet sind, lassen sich mit diesem Indikator meines Erachtens wichtige Strukturveränderungen der Ruhestandsmigration in Deutschland aufzeigen.

\section{Datenbasis, Methoden und erste Ergebnisse}

Zur Untersuchung des Strukturwandels der Ruhestandsmigration stütze ich mich im Folgenden auf Daten, die aus der INKAR-Datenbasis gewonnen wurden. ${ }^{5}$ Die Datenbasis enthält Aggregatdaten. Zu einem weiten Spektrum von demographischen, ökonomischen, sozialpolitischen sowie weiteren Sachverhalten enthält INKAR Indikatoren, die auf

\footnotetext{
${ }^{4}$ Ein Beispiel für weitere ähnliche Befunde aus Westdeutschland ist die Untersuchung von Schwarck (2008). Sie belegt für die Periode 2004-2006, dass die Generation 60+ in Baden-Württemberg die Verdichtungsräume im Saldo verlässt. Einzige Ausnahme mit im Saldo Zuwanderung dieser Altersgruppe ist Baden-Baden.

5 INKAR: Indikatoren und Karten zur Raum- und Stadtentwicklung, Ausgaben 2010-2017, herausgegeben vom Bundesinstitut für Bau-, Stadt- und Raumforschung (BBSR).
} 
der Basis unterschiedlicher Raumbezüge ${ }^{6}$ aggregiert wurden. Ausgegeben werden die Abfrageergebnisse in tabellarischer Form oder als Kartendarstellungen.

Wie bei anderen laufend fortgeschriebenen und aktualisierten Indikatorensystemen gibt es beim Versuch, längere Zeiträume zu analysieren, zum Teil Probleme, weil Änderungen am methodischen Konzept, an klassifikatorischen Abgrenzungen oder räumlichen Aggregationsabgrenzungen vorgenommen wurden. Dies trifft auch auf INKAR zu und hatte zur Folge, dass die folgenden Auswertungen sich auf unterschiedliche Versionen der Datenbasis beziehen. Der jeweils aktuellste Zeitbezug von INKAR ist beim hier betrachteten Indikator „Ältere Ruhestandswanderer“ (= Saldo der Zuzüge und Fortzüge pro 1.000 Einwohner der Altersgruppe 65+) das Jahr der Veröffentlichung minus drei. Die 2017 veröffentlichte Version enthält also als aktuellste Daten die Salden des Jahres 2014. Ein Problem war, dass die siedlungsstrukturelle Typologisierung im Hinblick auf Konzentration/Dekonzentration in den aktuellen Versionen verändert wurde. Eine differenziertere Kategorisierung wurde zugunsten einer gröberen aufgegeben. Da für meine Fragestellung die feine Untergliederung aussagekräftiger ist, verwende ich für den größten Teil der folgenden Analysen die INKAR-Ausgabe von 2015 (mit aktuellstem Zeitbezug: 2012). Bei anderen Auswertungen verwende ich die aktuellste Ausgabe von 2017 (mit aktuellstem Zeitbezug: 2014). ${ }^{7}$

Die im Folgenden betrachtete Aggregationsebene ist die „Kreisregion“. Auf dieser Ebene ist Deutschland in etwa 370 Regionen untergliedert, davon sind etwa 70 Fälle ausgewiesen als ,kreisfreie Großstädte“ (mehr als 100.000 Einwohner). Die genaue Anzahl der jeweils betrachteten Kreisregionen schwankt etwas, weil für bestimmte Zeitbezüge einige Kreisregionen wegen verzerrender Einflüsse ausgeschlossen werden mussten. ${ }^{8}$ Außerdem blieben über den betrachteten Zeitraum die Kreisregionen nicht identisch definiert. Teilweise verloren ehemals kreisfreie Großstädte diesen Status (z. B. Cottbus). Die Zahl der Großstädte ist in-

\footnotetext{
${ }^{6}$ Raumbezüge der aktuellen Version 2017 sind: Bund, West/Ost, Länder, NUTS-2-Deutschland, Raumordnungsregionen, Kreisregionen, Kreise, Gemeindeverbände, Regionstyp, Kreistyp, Arbeitsmarktregion, städtischer/ländlicher Raum, Stadt-/Gemeindetyp, IHK-Bezirke, Mittelbereiche, EU, NUTS 0, NUTS 1, NUTS 2. Einige dieser Raumbezüge unterscheiden dabei zusätzlich nach West- und Ostdeutschland.

7 Eine weitere wichtige Umstellung betrifft den Zugang zu INKAR. Bis 2015 war die Datenbasis als CD kostenpflichtig erhältlich, nunmehr ist die Nutzung nur noch online möglich. Damit wird die Nutzung zwar kostenfrei, die Extraktion von größeren Datenmengen, um sie mit Statistik-Software zu analysieren, ist aber schwieriger geworden.

${ }^{8}$ Beispielsweise gab es in den Regionen Unna, Göttingen, Osnabrück, Fürth und Rastatt große Erstaufnahmeeinrichtungen für durch Außenwanderung zugezogene Personen. Der Saldo von Fortzügen aus diesen Regionen war in den 1990er-Jahren extrem hoch, sodass bei Berechnungen diese Werte in diesen Perioden als fehlende Werte behandelt wurden.
}

folge der Bevölkerungsbestandsveränderungen und infolge von Gebietsstandsänderungen deshalb ebenfalls nicht gleich geblieben. Einige Großstädte sind in der Datei als solche nicht erkennbar, weil sie statistisch in Gemeindeverbänden enthalten sind (z. B. Hannover). Von den 2014 bestehenden 78 Großstädten in Deutschland sind nur 66 als „kreisfreie Großstadt" in der Datei separat ausgewiesen.

Der zentrale Indikator ist der Saldo der Zuzüge und Fortzüge bezogen auf 1.000 Einwohner der Altersgruppe $65+$ („Ältere Ruhestandswanderer“). Dieser Indikator erfasst nur die Wechsel des ersten Wohnsitzes; insofern unterschätzt er das Ausmaß der Ruhestandsmigration etwas. Nicht erfasst werden die temporäre Migration und multilokale Wohnformen, z. B. das Pendeln zwischen erstem und zweitem Wohnsitz. Zusätzlich wurde eine Reihe weiterer Indikatoren aus der INKAR-Datenbasis extrahiert, die geeignet erschienen, die Variation der Ruhestandsmigrationssalden zwischen den rund 370 Fällen im Rahmen von Regressionsmodellen zu erklären. Alle Daten wurden als SPSS-Datei konfiguriert und dann mit diesem Programmpaket analysiert.

Um zufällige Schwankungen auszugleichen, wurde nicht mit den Jahreswerten, sondern mit dem arithmetischen Mittel der Migrationssalden für folgende vier Perioden gerechnet: 1995-1999, 2000-2004, 2005-2009 und 2010-2012. Die Entwicklung jeder der rund 370 Kreisregionen kann so übersichtlich in vier Werten dargestellt werden. Im Hinblick auf die Frage der Reurbanisierung ist die (bis zur Ausgabe 2015) in INKAR enthaltene Regional-Typologie gut verwendbar. Die ursprüngliche Unterscheidung von neun Kreistypen wurde, teils wegen zu geringer Besetzungszahlen, teils um die hier interessierende Kernstadt/Nicht-Kernstadt-Unterscheidung klarer zur Geltung kommen zu lassen, auf eine Typologie von sechs Regionstypen reduziert: ${ }^{9}$

- Typ 1: Kernstadt in Agglomeration

- Typ 2: Hochverdichtete Kreisregion in Agglomeration

- Typ 3: Weniger verdichtete Kreisregion in Agglomeration

- Typ 4: Kernstadt im verstädterten Raum

- Typ 5: Weniger verdichtete Kreisregion im verstädterten Raum

- Typ 6: Kreisregion im ländlichen Raum

Etwas pauschalierend kann im Rahmen dieses methodischen Ansatzes das alte Muster der Ruhestandsmigration in zwei Sätzen zusammengefasst werden: Die Typen 1 und 4 haben mehr Fort- als Zuzüge zu verzeichnen und die Typen

\footnotetext{
9 Zusammengefasst wurden: „Verdichteter Kreis in Agglomeration“ mit „Ländlicher Kreis in Agglomeration“, „Verdichteter Kreis im verstädterten Raum“ mit „Ländlicher Kreis im verstädtertem Raum“ sowie „Ländlicher Kreis höherer Dichte im ländlichen Raum“ mit „Ländlicher Kreis geringer Dichte im ländlichen Raum“.
} 
Abbildung 1 Saldo der Zuzüge minus Fortzüge (Altersgruppe $65+$ ) bezogen auf 1.000 Personen dieser Altersgruppe in rund 370 Kreisregionen

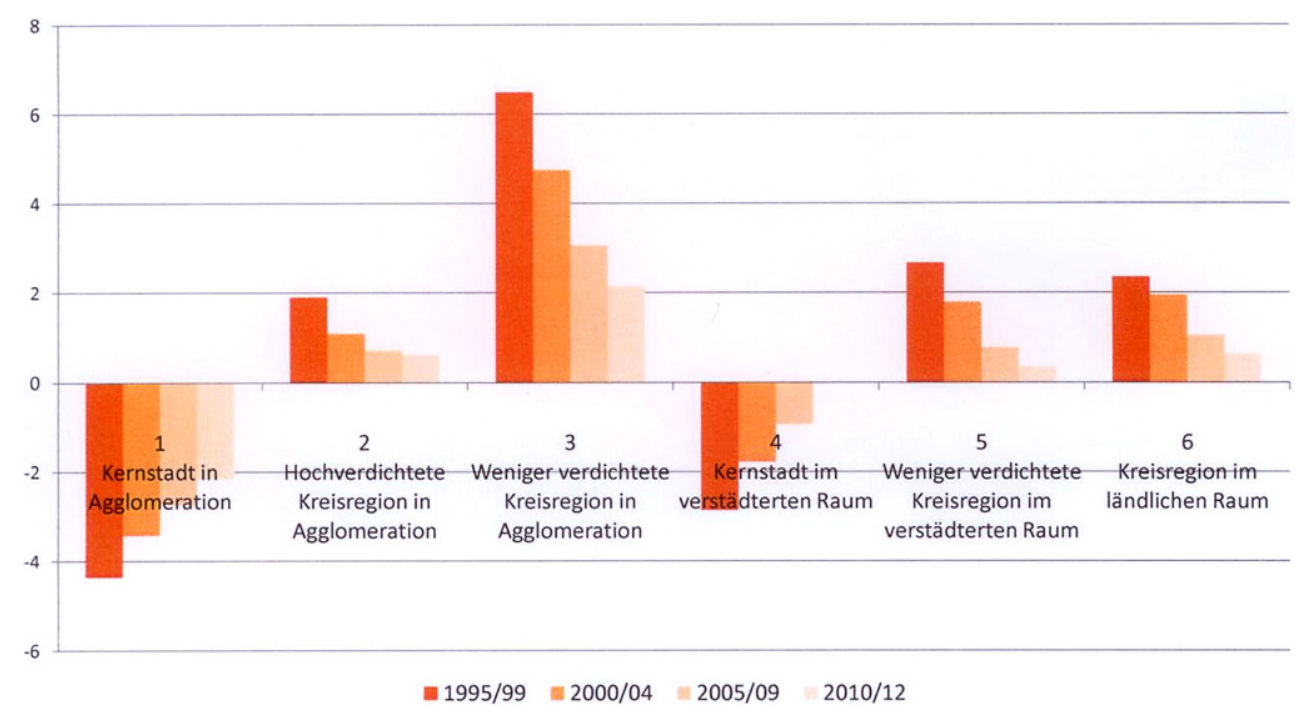

2, 3, 5 und 6 stehen auf der ,Gewinnerseite ' mit einem unterschiedlich hoch ausfallenden positiven Saldo. Abbildung 1 und Tabelle 1 zeigen die Salden für die vier Perioden. Das bekannte Muster der „Stadtflucht“ der Älteren ist 19951999 noch deutlich sichtbar. Der suburbane Raum und die ländlichen Gebiete sind die typischen Zielregionen der Ruhestandswanderung. Ganz ähnlich ist die Situation, wenn man die Periode 2000-2004 betrachtet. Allerdings sind für alle Regionstypen die Saldenwerte deutlich geringer. Die Neigung zum Wohnsitzwechsel in weniger konzentrierte Siedlungsformen ist offenbar zurückgegangen.

\section{Das alte Muster der Ruhestandsmigration schwächt sich ab}

Betrachtet man alle vier Perioden insgesamt, dann zeigt sich tatsächlich ein Trend. Die Verluste der Städte werden ebenso wie die Zugewinne des Umlands und der peripheren Regionen immer geringer. In dieser Hinsicht trägt die Ruhestandsmigration nahezu nichts mehr zu Bevölkerungsumschichtungen bei. Berücksichtigt man zusätzlich, dass in der Periode von 1995 bis 2005 das Volumen der Binnenwanderung der 65-Jährigen deutlich zurückgegangen ist (Friedrich 2008: 186 f.), dann wird man die Senioren schwerlich zu den Bevölkerungsgruppen zählen können, die immer mobiler werden.

Um etwas näher zu explorieren, welche Merkmale die Kreisregionen mit Zuwanderungsgewinnen von den Regionen unterscheiden, aus denen die Generation 65+ im Saldo abwandert, wurde eine OLS-Regression berechnet. Als unabhängige Variablen wurden dabei Indikatoren eingeführt, die sich in vorab durchgeführten OLS-Regressionen als besonders gewichtige Einflussfaktoren bei der Erklärung der
Varianz der Migrationssalden erwiesen hatten. ${ }^{10} \mathrm{Um}$ ein vollständiges Bild zu erhalten, wäre es eigentlich erforderlich gewesen, für jeden der vier Zeiträume eine multiple Regression durchzuführen. Da diese Analyse jedoch nur als ergänzende Information dafür dienen soll, wie das traditionelle Muster der Ruhestandsmigration in Aggregatdatenanalysen zum Ausdruck kommt, wurde eine Periode exemplarisch herausgegriffen. Die aktuellste Periode bot sich dazu nicht an, da die Streubreite der Migrationssalden als $\mathrm{zu}$ gering erschien. Aus diesem Grund wurde die zweitaktuellste Periode gewählt. Sie wurde auch deshalb gewählt, weil sie vielleicht ,normaler' ist als die vorhergehenden Perioden, in denen möglicherweise noch Sondereinflüsse im Zusammenhang mit nachholenden Entwicklungen im Zuge der Wiedervereinigung wirksam waren (z. B. der Suburbanisierungsschub in Ostdeutschland in den 1990er-Jahren).

Zwei Gruppen von unabhängigen Variablen wurden ausgewählt, um das Muster der Einflüsse zu beschreiben: zum einen Push-Faktoren, die zur Abwanderung der Älteren führen, zum anderen Pull-Faktoren, also Anreize, die Zielregionen bieten. $\mathrm{Zu}$ den ersteren zählt das Vermeiden hochkonzentrierter Siedlungsformen (gemessen mit dem Indikator „Einwohnerdichte“), die Tendenz, sozialstrukturell schwächere Regionen (gemessen mit dem Indikator „Ausmaß der Altersarmut“) aber auch ,teure“

\footnotetext{
${ }^{10}$ Es wurden verschiedene Indikatoren, von denen vermutet werden kann, dass sie mit den Salden der Ruhestandsmigration korrelieren (vgl. die synoptische Zusammenstellung aller aus vorliegenden Studien bekannten Einflussvariablen bei Walters 1994), in OLS-Regressionen ,getestet", und diejenigen mit den größten Varianzaufklärungsbeiträgen wurden in die hier berichtete Gleichung aufgenommen. Signifikanzberechnungen spielten dabei keine Rolle, da es sich im vorliegenden Fall um eine deskriptive Aufbereitung von Parametern der Grundgesamtheit handelt und nicht um eine Stichprobe. OLS: Ordinary-LeastSquares-Model (Schätzmodell).
} 
Tabelle 1 Saldo der Zuzüge minus Fortzüge (Altersgruppe 65+) bezogen auf 1.000 Personen dieser Altersgruppe in rund 370 Kreisregionen (in Klammern: Zahl der Regionstypen)

\begin{tabular}{|c|c|c|c|c|}
\hline & 1995-1999 & 2000-2004 & 2005-2009 & 2010-2012 \\
\hline Typ 1: Kernstadt in Agglomeration & $\begin{array}{l}-4.36 \\
(41)\end{array}$ & $\begin{array}{l}-3.42 \\
(41)\end{array}$ & $\begin{array}{l}-2.74 \\
(41)\end{array}$ & $\begin{array}{l}-2.15 \\
(41)\end{array}$ \\
\hline Typ 2: Hochverdichtete Kreisregion in Agglomeration & $\begin{array}{l}1.90 \\
(38)\end{array}$ & $\begin{array}{l}1.08 \\
(39)\end{array}$ & $\begin{array}{l}0.71 \\
(39)\end{array}$ & $\begin{array}{l}0.62 \\
(38)\end{array}$ \\
\hline Typ 3: Weniger verdichtete Kreisregion in Agglomeration & $\begin{array}{l}6.49 \\
(47)\end{array}$ & $\begin{array}{l}4.75 \\
(47)\end{array}$ & $\begin{array}{l}3.06 \\
(47)\end{array}$ & $\begin{array}{l}2.15 \\
(47)\end{array}$ \\
\hline Typ 4: Kernstadt im verstädterten Raum & $\begin{array}{l}-2.86 \\
(29)\end{array}$ & $\begin{array}{l}-1.77 \\
(29)\end{array}$ & $\begin{array}{l}-0.94 \\
(29)\end{array}$ & $\begin{array}{l}0.01 \\
(27)\end{array}$ \\
\hline Typ 5: Weniger verdichtete Kreisregion im verstädterten Raum & $\begin{array}{l}2.67 \\
(136)\end{array}$ & $\begin{array}{l}1.79 \\
(138)\end{array}$ & $\begin{array}{l}0.77 \\
(138)\end{array}$ & $\begin{array}{l}0.35 \\
(138)\end{array}$ \\
\hline \multirow[t]{2}{*}{ Typ 6: Kreisregion im ländlichen Raum } & $\begin{array}{l}2.35 \\
(74)\end{array}$ & $\begin{array}{l}1.94 \\
(75)\end{array}$ & $\begin{array}{l}1.05 \\
(75)\end{array}$ & $\begin{array}{l}0.63 \\
(75)\end{array}$ \\
\hline & $\mathrm{N}=365$ & $\mathrm{~N}=369$ & $\mathrm{~N}=369$ & $\mathrm{~N}=366$ \\
\hline
\end{tabular}

und wirtschaftlich prosperierende Regionen (gemessen mit den Indikatoren „Baulandpreise“ und „Gewerbesteueraufkommen“) zu verlassen. Als letztes zählt dazu die Abwanderung aus (alt)industriellen Regionen (gemessen mit dem Indikator „Industriequote“). Als Pull-Faktoren wurden berücksichtigt: die Verfügbarkeit attraktiver, neuer Wohnungen (gemessen mit dem Indikator „Neubauwohnungen pro Einwohner") und die Eignung einer Region als Urlaubsdestination (gemessen mit dem Indikator „Durchschnittliche Verweildauer in Fremdenverkehrsbetrieben“). Zusätzlich wurde der Indikator „Bevölkerungsentwicklung 2004/2005“ einbezogen. Er fasst alle Pull-Faktoren zusammen, die eine Region für die Bevölkerung insgesamt attraktiv machen, wenn die oben genannten Einflussgrößen kontrolliert sind. Die resultierende Gleichung lautet: ${ }^{11}$

Geschätztes Saldo der Ruhestandsmigration =

Konstante

$+b_{1} *$ Bevölkerungsentwicklung (2004/2005)

$+b_{2} *$ Neubauwohnungen pro Einwohner (2004/2008)

$+b_{3} *$ Durchschnittliche Verweildauer

in Fremdenverkehrsbetrieben (2009)

$+b_{4} *$ Einwohnerdichte $(2004 / 2008)$

$+b_{5} *$ Baulandpreise $(2004 / 2008)$

$+b_{6} *$ Gewerbesteuer (2004/2008)

$+b_{7} *$ Ausmaß der Altersarmut (2009)

$+b_{8} *$ Industriequote (2008)

+ Residuum

11 Teilweise sind die Indikatoren für jedes Jahr der Periode im Datensatz vorhanden; dann wurde das arithmetische Mittel verwendet. Teilweise ist der Indikator nur für ein einzelnes Jahr verfügbar. Die Multikollinearität der unabhängigen Variablen ist nicht so stark, dass ein oder mehrere Prädiktoren hätten ausgeschlossen werden müssen.
Tabelle 2 zeigt die bivariaten Korrelationen aller Variablen.

Die acht einbezogenen Variablen erklären $65 \%$ der Varianz der Ruhestandsmigrationssalden (vgl. Tabelle 3). Zum Anstieg der Salden kommt es besonders in den Regionen, in denen die Bevölkerung insgesamt zunimmt. Menschen im Ruhestand ziehen also tendenziell dorthin, wohin auch andere Bevölkerungsgruppen ziehen. Teilweise sicher auch deshalb, um weiterhin in der Nähe von Kindern leben zu können, die nach einer Familiengründung umziehen. Zum Anstieg der Ruhestandsmigration tragen außerdem zwei weitere Faktoren mit positiven Regressionsgewichten bei: zum einen die Neubautätigkeit in den Zielregionen, zum anderen die touristische Attraktivität der Region. Alle anderen einbezogenen Variablen haben negative Regressionsgewichte. Verluste bei der Bevölkerungsgruppe der Ruheständler sind zu erwarten mit dem Anstieg der Einwohnerdichte in einer Region, bei höheren Anteilen von Älteren, die auf Grundsicherung angewiesen sind, bei stärker industriell geprägter Wirtschaftsstruktur, bei höheren Baulandpreisen sowie bei hohem Gewerbesteueraufkommen.

Zur Beantwortung der Frage, ob die Ruhestandsmigration zur Reurbanisierung beiträgt oder nicht, sind die bisher gezeigten Befunde nur ein erster Schritt. Zwar gingen die Negativsalden der Kernstädte in Agglomerationen und der Kernstädte im verstädterten Raum drastisch zurück, aber hinter diesen Durchschnittswerten stehen ja möglicherweise ganz unterschiedliche Entwicklungen in den einzelnen Städten. Zu diesem Zweck werfe ich einen Blick auf die 66 Großstädte mit mehr als 100.000 Einwohnern, die in der 2017-Version von INKAR als kreisfreie Großstädte ausgewiesen sind. Die folgenden Analysen beziehen sich also auf eine geringfügig von Abbildung 1 abweichend abgegrenzte Gruppe von Städten. Die letzte Periode reicht bis zum Jahr 2014. Einige wenige schrumpfende Städte sind aus dieser Gruppe ausgeschieden, eine etwas größere Zahl 
Tabelle 2 Bivariate Produkt-Moment-Korrelationen der im Modell einbezogenen Variablen

\begin{tabular}{|c|c|c|c|c|c|c|c|c|}
\hline & $\begin{array}{l}\text { Migrationssaldo } \\
2005 / 2009\end{array}$ & $\begin{array}{l}\text { Bevölkerungs- } \\
\text { entwicklung }\end{array}$ & $\begin{array}{l}\text { Bauland- } \\
\text { preise }\end{array}$ & $\begin{array}{l}\text { Einwohner- } \\
\text { dichte }\end{array}$ & $\begin{array}{l}\text { Gewerbe- } \\
\text { steuer }\end{array}$ & $\begin{array}{l}\text { Neubauwohnungen } \\
\text { pro Einwohner }\end{array}$ & $\begin{array}{l}\text { Verweil- } \\
\text { dauer } \\
\text { Hotel }\end{array}$ & $\begin{array}{l}\text { Industrie- } \\
\text { quote }\end{array}$ \\
\hline $\begin{array}{l}\text { Bevölkerungs- } \\
\text { entwicklung }\end{array}$ & 0,26 & --- & & & & & & \\
\hline Baulandpreise & $-0,23$ & 0,62 & --- & & & & & \\
\hline Einwohnerdichte & $-0,52$ & 0,36 & 0,63 & --- & & & & \\
\hline Gewerbesteuer & $-0,37$ & 0,49 & 0,70 & 0,55 & --- & & & \\
\hline $\begin{array}{l}\text { Neubauwohnungen } \\
\text { pro Einwohner }\end{array}$ & 0,52 & 0,61 & 0,26 & $-0,17$ & 0,12 & --- & & \\
\hline $\begin{array}{l}\text { Verweildauer } \\
\text { Hotel }\end{array}$ & 0,27 & $-0,27$ & $-0,34$ & $-0,41$ & $-0,34$ & 0,01 & --- & \\
\hline Industriequote & $-0,20$ & 0,00 & 0,01 & $-0,05$ & 0,28 & 0,03 & $-0,08$ & --- \\
\hline $\begin{array}{l}\text { Grundsicherung } \\
\text { Alter }\end{array}$ & $-0,41$ & 0,41 & 0,52 & 0,73 & 0,58 & $-0,07$ & $-0,27$ & $-0,32$ \\
\hline
\end{tabular}

Tabelle 3 Koeffizienten der multiplen Regression der durchschnittlichen Ruhestandsmigrationssalden (2004/2009) (370 Kreisregionen)

\begin{tabular}{|c|c|c|c|c|}
\hline & $\begin{array}{l}\text { Regressions- } \\
\text { koeffizient }\end{array}$ & Standardfehler & $\begin{array}{l}\text { Standard } \\
\text { Regressionskoeffizient }\end{array}$ & $\mathrm{T}$ \\
\hline (Konstante) & 3,22 & 0,55 & & 5,81 \\
\hline Bevölkerungsentwicklung 2004/2005 & 0,499 & 0,05 & 0,55 & 10,18 \\
\hline $\begin{array}{l}\text { Neubauwohnungen pro Einwohner } \\
(2004 / 2008)\end{array}$ & 0,433 & 0,11 & 0,19 & 3,90 \\
\hline $\begin{array}{l}\text { Durchschnittliche Verweildauer in Fremden- } \\
\text { verkehrsbetrieben (2009) }\end{array}$ & 0,284 & 0,09 & 0,11 & 3,11 \\
\hline Einwohnerdichte $(2004 / 2008)$ & $-0,001$ & 0,00 & $-0,32$ & $-5,54$ \\
\hline Ausmaß der Altersarmut (2009) & $-0,470$ & 0,12 & $-0,19$ & $-3,83$ \\
\hline Industriequote (2008) & $-0,059$ & 0,01 & $-0,17$ & $-4,87$ \\
\hline Baulandpreise (2004/2008) & $-0,003$ & 0,00 & $-0,15$ & $-2,67$ \\
\hline Gewerbesteuer (2004/2008) & $-0,002$ & 0,00 & $-0,19$ & $-3,57$ \\
\hline
\end{tabular}

$\mathrm{R}=0,81, \mathrm{R}^{2}=0,65$, korrigiertes $\mathrm{R}^{2}=0,64$

von Großstädten ist nicht als „kreisfreie Großstadt“ in der Datei enthalten, weil deren Wert nicht von den Werten der Gemeindeverbünde, denen sie angehören, getrennt werden kann. Insgesamt fehlen zwölf Städte, die größte davon ist Hannover. Die Fragen lauten: Gibt es im Zeitverlauf immer mehr Städte, in denen tatsächlich ein Trendwechsel zu beobachten ist? Und: Wo gibt es einen Überschuss der Zuzüge der Generation 65+?

Die Antwort findet sich in den Abbildungen 2, 3, 4 und 5. Ende der 1990er-Jahre gab es nur eine Handvoll unter den 66 betrachteten Großstädten, die einen - zumeist sehr kleinen - Positivsaldo aufzuweisen hatten. Auf der anderen Seite gab es sehr viele Städte mit einem hohen Negativsaldo. In den folgenden Perioden steigt die Zahl der Großstädte mit einem Zuwanderungsüberschuss dann beständig an. Die Negativsalden der ,Verliererstädte' werden immer geringer und es tauchen erste Großstädte auf, die einen nennenswer- ten Positivsaldo aufzuweisen haben. Besonders deutlich ist diese Zuwanderung in Potsdam $(+4,98) .{ }^{12}$

Die Städte mit den höchsten Abwanderungstendenzen sind nach wie vor die Kernstädte in den westdeutschen Metropolregionen. München, Offenbach, Frankfurt und Stuttgart landen hier regelmäßig auf vorderen Plätzen. Andere Städte sind von einem Extrem zum anderen gewechselt. So war Leipzig in der Periode 1995-1999 die Stadt mit dem größten Negativsaldo; 2010-2014 gehört Leipzig zu den Städten mit deutlichem Zuzugsüberschuss. Die den Abbildungen 2, 3, 4 und 5, die das Muster des Wandels sichtbar machen sollen, zugrundeliegenden Werte zeigt im Einzelnen Tabelle 4. Es ist natürlich nicht möglich, hier auf die Entwicklung einzelner Städte genauer einzugehen. Auffällig ist aber, dass die meisten Städte mit einem $\mathrm{Zu}$ -

\footnotetext{
12 Ähnlich hoch war der Positivsaldo 2004/2009 auch in der Stadt Cottbus. Die bis 2012 noch als kreisfreie Großstadt ausgewiesene Stadt gehört heute nicht mehr zu dieser Gruppe, sondern wird nur noch zusammen mit ihrem Umland ausgewiesen.
} 
Abbildung 2 Ruhestandsmigrationssalden von 66 kreisfreien Großstädten ( $\varnothing$ 19951999)

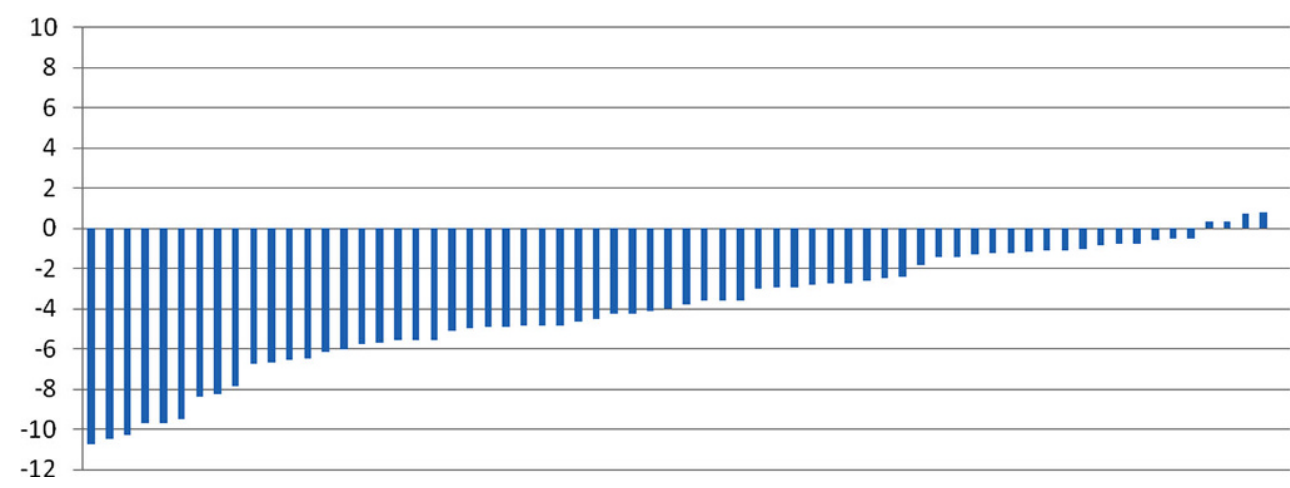

Abbildung 3 Ruhestandsmigrationssalden von 66 kreisfreien Großstädten $(\varnothing 2000$ 2004)

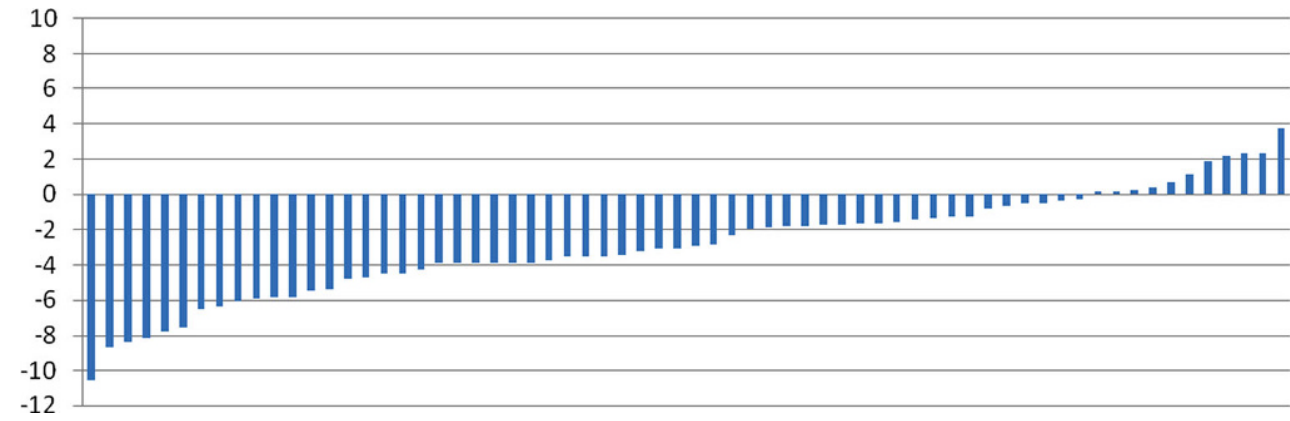

wanderungsüberschuss in Ostdeutschland liegen. Von den elf Großstädten, die 2010-2014 Positivsalden von größer als 1,00 haben, liegen sieben in Ostdeutschland. Potsdam ragt mit einem Saldo von 7,24 deutlich heraus. Positivsalden von 1,34 bis 2,46 haben im Westen nur Oldenburg, Fürth, Koblenz und Lübeck.

Insgesamt scheinen die Veränderungen in den Salden im ersten Zehnjahreszeitraum größer gewesen $\mathrm{zu}$ sein als die Veränderungen in den beiden Perioden des zweiten Zehnjahreszeitraums. Besonders von der ersten auf die dritte Periode haben einige Städte ihren Platz in der Rangordnung stark verändert. Um diesen Wandel in einzelnen Städten zu veranschaulichen, sind in Abbildung 6 die Salden der Periode 1995-1999 gegen die Salden der Periode 2005-2009 dargestellt. ${ }^{13}$

Die Abbildung 6 verdeutlicht, dass es eine Gruppe von westdeutschen Metropolen und altindustriellen Großstädten gibt, die im gesamten Zeitraum Abwanderungsüberschüsse bei den Senioren aufzuweisen haben. Und es gibt auf der anderen Seite die ostdeutschen Großstädte, die - ganz gleich, wo sie in der Periode 1995-1999 standen - heute Zugewinne durch die Altenwanderung zu verzeichnen haben. Dieser für die Ruhestandsmigration zu konstatierende Tatbestand, eher Stabilität im Westen und eher Veränderung im Osten, wird von Milbert (2017: 4) ganz generell als ein

$\overline{13}$ Die Fallzahl beträgt hier 70, weil das die Zahl von Großstädten war, die zum Ende der Periode 2005-2009 in INKAR als „kreisfreie Großstadt" ausgewiesen war.
Kennzeichen der Reurbanisierung in Deutschland herausgestellt: „In Ostdeutschland kann ein deutlicher Reurbanisierungsprozess seit 1989, dem Beginn der Datenreihe, beobachtet werden - mit nur einer kurzen Abschwächungsoder Verzögerungsphase in den Nachwendejahren. Die ostdeutschen Agglomerationen haben in den letzten 26 Jahren über vier Prozentpunkte gewonnen. Ohne Berlin wächst ihre Bedeutung sogar um sieben Prozentpunkte. Verglichen mit den ostdeutschen Entwicklungen verschieben sich die Bedeutungen der westdeutschen städtischen Regionen sehr moderat". ${ }^{14}$ Angesichts der hier vorgestellten Indikatoren kann ergänzend hinzugefügt werden, dass die Seniorengeneration, wenn auch sehr moderat, aktiv zu dieser Reurbanisierung beiträgt.

\section{Diskussion und Schlussfolgerungen}

Ausgangspunkt der Untersuchung war die Frage, ob sich in Deutschland bei der Ruhestandsmigration das alte Muster der counterurbanisation (und auch der Suburbanisierung) beobachten lässt oder ob es auch bei der älteren Generation eine Trendwende hin zur Reurbanisierung gibt. Die Auswertung der INKAR-Daten zeigt, dass die Frage dif-

\footnotetext{
${ }^{14}$ Es ist allerdings unklar, inwieweit bei der Analyse der Veränderungen die im Osten viel stärker zu Buche schlagenden Gebietsstandsänderungen berücksichtigt worden sind (vgl. die Kritik von Kauffmann 2015).
} 
Abbildung 4 Ruhestandsmigrationssalden von 66 kreisfreien Großstädten ( $\varnothing 2005$ 2009)
Abbildung 5 Ruhestandsmigrationssalden von 66 kreisfreien Großstädten ( $\varnothing$ 20102014)
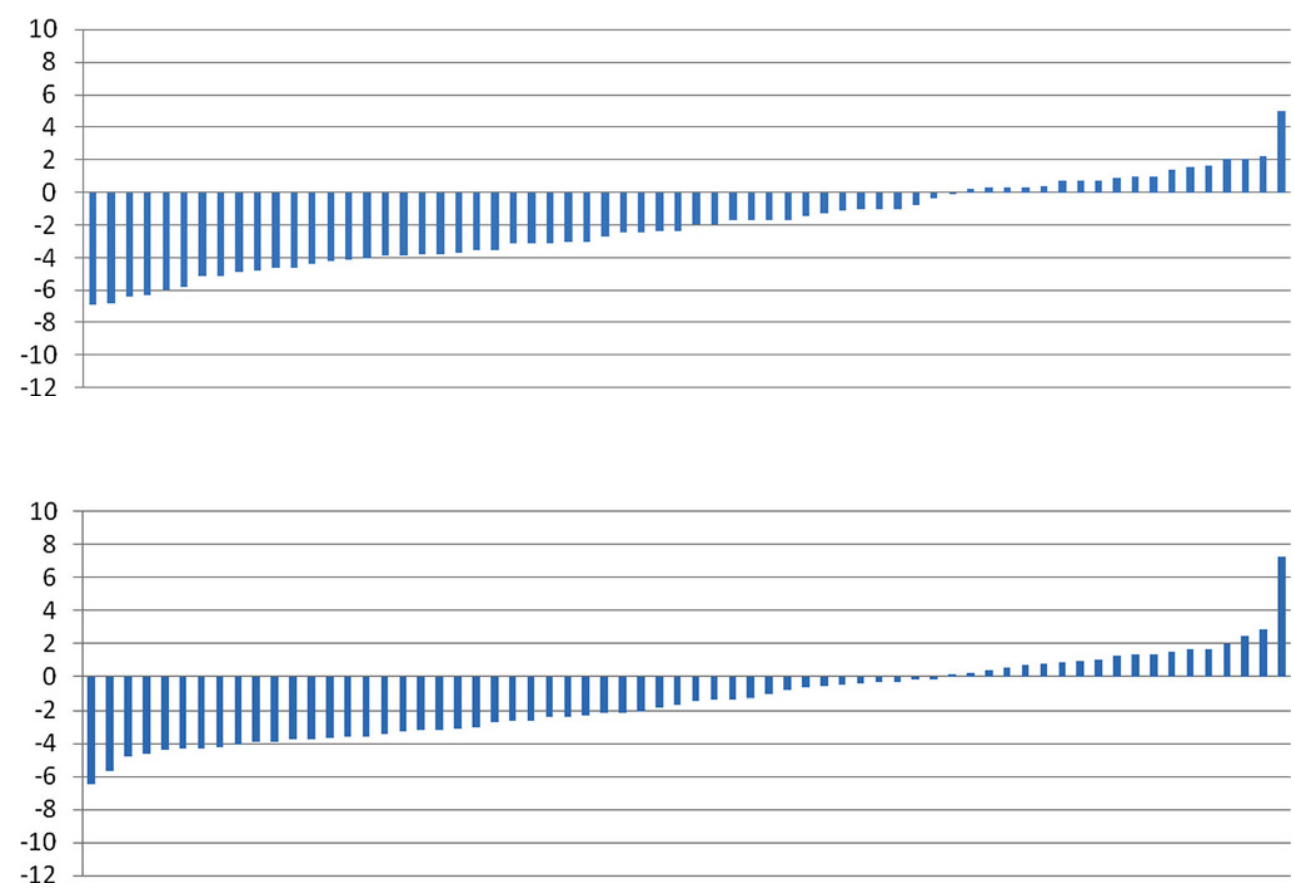

ferenziert zu beantworten ist. Insgesamt gehen die Verluste der Kernstädte in Agglomerationen und in verstädterten Regionen tatsächlich deutlich zurück auf ein Niveau, das so gering ist, dass regionale Bevölkerungsumschichtungen bei den Senioren in dieser Hinsicht kaum noch stattfinden. Die Betrachtung der kreisfreien Großstädte zeigt aber, das Reurbanisierung (wenn man das so nennen will) in zwei Formen auftritt: Nur in der Minderheit der Städte ist ein Vorzeichenwechsel der Saldoraten der Ruhestandsmigration zu verzeichnen, und ganz offensichtlich ist diese Nettozuwanderung der Älteren in die Städte im Osten wesentlich ausgeprägter als im Westen der Bundesrepublik. In der Mehrzahl der Großstädte im Westen ist das alte Muster noch sichtbar, aber es lässt sich ein deutlicher Rückgang der Wanderungsverluste feststellen.

Wie lassen sich die Befunde interpretieren? Ich betrachte zunächst die Situation im Hinblick auf die im suburbanen Umland lebende Bevölkerung. In diesem Siedlungstyp findet ein Großteil des gesellschaftlichen Alterungsprozesses statt (vgl. Nuissl/Bigalke 2007). Im Westen setzte der Suburbanisierungsprozess schon in den 1960er-Jahren ein. Im Osten gab es „eine gleichsam ,aus dem Nichts“ entstandene und ab etwa 1993 rasant anwachsende ,HyperSuburbanisierung', die bis Ende der 1990er-Jahre wieder abflacht“ (Köppen/Mai/Schlömer 2007: 217). Häufig war der Umzug ins Umland dabei mit der Bildung von Wohneigentum verbunden. Frühe Prognosen einer Rückkehr der Senioren in die Stadt haben vor allem auf das große Potenzial bei den in Suburbia Wohnenden hingewiesen (vgl. Opaschowski 2005). Allerdings haben empirische Untersuchungen bislang diese Erwartungen nicht bestätigt. „Zwar ist es unstrittig, dass die meisten Wohnungen nach dem Auszug der Kinder aus dem Elternhaus ,zu groß ‘ für die verbleibenden Bewohner sind. Ebenso erwiesen ist es aber, dass dieses Missverhältnis offenbar kaum zum Umzugswillen beiträgt. Vielmehr sind gerade bei den Älteren Remanenzeffekte nachweisbar, d.h. eine Zunahme der Wohnfläche durch Auszug der Kinder und schließlich den Tod des Partners. Nicht etwa der freiwillige Umzug im Alter, sondern ,Ageing in Place ' bestimmt (noch) das durchschnittliche Verhalten“ (Köppen/Mai/Schlömer 2007: 225). Neuere Untersuchungen haben diese Präferenz für das Wohnenbleiben in Suburbia für verschiedene Regionen nachgewiesen. Glasze und Graze (2007) zeigen dies für Mainz, Kramer und Pfaffenbach $(2009,2011)$ stellen bei Untersuchungen in der Stadt und im Umland von München, Aachen und Karlsruhe die gleiche Vorliebe für das Verbleiben am Ort fest: „Durch fast alle qualitativen Interviews zog sich als roter Faden, dass für die meisten Befragten ihre jeweilige Wohngemeinde der ideale Wohnort ist und das derzeitige Wohnviertel deutlich mehr Vorteile als Nachteile besitzt" (Kramer/Pfaffenbach 2011: 86). ${ }^{15}$

Vor dem Hintergrund solcher Untersuchungen ist es eher unwahrscheinlich, dass die Nettowanderungsgewinne in ostdeutschen Großstädten ausgerechnet auf die schnelle Rückkehr der spät Suburbanisierten zurückzuführen sein sollten. Viel plausibler ist die Annahme, das es sich hierbei teils um eine Rückwanderung von Personen handelt, die

\footnotetext{
15 Die Aussage bezieht sich nicht nur auf die im Umland lebende Bevölkerung, sondern auch auf die in kernstädtischen Vierteln Wohnenden.
} 
Tabelle 4 Salden der Zuzüge und Fortzüge 65+ (je 1.000 Einwohner) für 66 kreisfreie Großstädte

\begin{tabular}{|c|c|c|c|c|c|}
\hline & $\begin{array}{l}\text { Kreisfreie } \\
\text { Großstadt } \\
2014\end{array}$ & $\begin{array}{l}\text { Saldo } \\
1995- \\
1999\end{array}$ & $\begin{array}{l}\text { Saldo } \\
2000- \\
2004\end{array}$ & $\begin{array}{l}\text { Saldo } \\
2005- \\
2009\end{array}$ & $\begin{array}{l}\text { Saldo } \\
2010- \\
2014\end{array}$ \\
\hline 1 & Kiel & $-9,68$ & $-7,76$ & $-3,10$ & $-0,14$ \\
\hline 2 & Lübeck & $-1,12$ & $-1,66$ & 0,98 & 2,46 \\
\hline 3 & Hamburg & $-5,58$ & $-5,42$ & $-4,22$ & $-3,72$ \\
\hline 4 & Braunschweig & $-4,92$ & $-5,86$ & $-1,94$ & $-1,36$ \\
\hline 5 & Wolfsburg & $-1,08$ & $-3,10$ & $-3,68$ & $-3,26$ \\
\hline 6 & Oldenburg & 0,76 & 0,14 & 0,68 & 1,34 \\
\hline 7 & Osnabrück & $-4,86$ & $-6,08$ & $-4,40$ & $-4,36$ \\
\hline 8 & Bremen & $-4,96$ & $-3,86$ & $-2,42$ & $-2,14$ \\
\hline 9 & Bremerhaven & $-9,70$ & $-8,42$ & $-6,02$ & $-3,56$ \\
\hline 10 & Düsseldorf & $-6,46$ & $-5,86$ & $-5,16$ & $-4,62$ \\
\hline 11 & Duisburg & $-6,66$ & $-5,9$ & $-5,16$ & $-3,64$ \\
\hline 12 & Essen & $-4,14$ & $-3,04$ & $-3,16$ & $-2,28$ \\
\hline 13 & Krefeld & $-4,26$ & $-3,42$ & $-1,68$ & $-1,84$ \\
\hline 14 & $\begin{array}{l}\text { Mönchenglad- } \\
\text { bach }\end{array}$ & $-0,84$ & $-0,78$ & $-1,74$ & $-1,00$ \\
\hline 15 & $\begin{array}{l}\text { Mülheim } \\
\text { Ruhr }\end{array}$ & $-1,04$ & $-1,64$ & $-2,7$ & $-1,26$ \\
\hline 16 & Oberhausen & $-3,58$ & $-3,54$ & $-1,92$ & $-1,42$ \\
\hline 17 & Remscheid & $-4,66$ & $-4,80$ & $-4,80$ & $-3,90$ \\
\hline 18 & Solingen & $-1,14$ & 0,42 & 0,74 & 0,26 \\
\hline 19 & Wuppertal & $-4,84$ & $-3,86$ & $-3,88$ & $-3,62$ \\
\hline 20 & Bonn & $-2,58$ & $-1,24$ & $-1,28$ & $-0,16$ \\
\hline 21 & Köln & $-5,54$ & $-4,52$ & $-3,76$ & $-3,18$ \\
\hline 22 & Leverkusen & $-1,84$ & $-1,42$ & $-2,38$ & $-2,40$ \\
\hline 23 & Bottrop & $-0,74$ & $-0,52$ & 0,30 & $-0,28$ \\
\hline 24 & Gelsenkirchen & $-5,08$ & $-4,26$ & $-5,82$ & $-4,32$ \\
\hline 25 & Münster & $-4,80$ & $-4,50$ & $-0,78$ & 0,38 \\
\hline 26 & Bielefeld & $-3,56$ & $-3,92$ & $-4,16$ & $-3,44$ \\
\hline 27 & Bochum & $-3,60$ & $-3,92$ & $-3,54$ & $-3,74$ \\
\hline 28 & Dortmund & $-6,54$ & $-3,88$ & $-3,08$ & $-2,70$ \\
\hline 29 & Hagen & $-8,26$ & $-7,56$ & $-4,9$ & $-4,28$ \\
\hline 30 & Hamm & $-5,56$ & $-2,92$ & $-1,42$ & $-0,78$ \\
\hline 31 & Herne & $-4,88$ & $-3,24$ & $-2,50$ & $-1,34$ \\
\hline 32 & Darmstadt & $-3,80$ & $-1,96$ & $-1,74$ & $-0,66$ \\
\hline 33 & $\begin{array}{l}\text { Frankfurt am } \\
\text { Main }\end{array}$ & $-10,28$ & $-8,66$ & $-6,34$ & $-4,82$ \\
\hline 34 & Offenbach & $-10,50$ & $-10,56$ & $-6,92$ & $-5,66$ \\
\hline 35 & Wiesbaden & $-2,92$ & $-3,86$ & $-3,76$ & $-3,10$ \\
\hline 36 & Kassel & $-6,74$ & $-6,05$ & $-4,68$ & $-4,20$ \\
\hline 37 & Koblenz & $-2,42$ & $-1,24$ & $-1,08$ & 1,66 \\
\hline 38 & Trier & $-6,00$ & $-3,48$ & $-3,92$ & $-3,92$ \\
\hline 39 & Ludwigshafen & $-5,74$ & $-5,48$ & $-4,02$ & $-2,62$ \\
\hline 40 & Mainz & $-2,48$ & $-1,36$ & 0,32 & $-1,66$ \\
\hline 41 & Stuttgart & $-8,36$ & $-6,4$ & $-6,40$ & $-4,04$ \\
\hline 42 & Heilbronn & $-2,78$ & $-2,84$ & $-3,06$ & $-2,10$ \\
\hline 43 & Karlsruhe & $-1,40$ & $-1,68$ & $-3,52$ & $-3,18$ \\
\hline 44 & Heidelberg & $-3,02$ & $-3,50$ & $-3,16$ & $-2,36$ \\
\hline 45 & Mannheim & $-4,24$ & $-4,68$ & $-4,68$ & $-3,02$ \\
\hline
\end{tabular}

Tabelle 4 Salden der Zuzüge und Fortzüge 65+ (je 1.000 Einwohner) für 66 kreisfreie Großstädte (Fortsetzung)

\begin{tabular}{llllll}
\hline 46 & Pforzheim & 0,32 & $-0,54$ & 0,76 & $-0,40$ \\
47 & Freiburg & $-1,20$ & 0,18 & 1,00 & 0,96 \\
48 & Ulm & $-0,76$ & $-1,84$ & $-2,44$ & 0,20 \\
49 & Ingolstadt & 0,84 & 0,68 & 1,52 & $-0,30$ \\
50 & München & $-7,88$ & $-8,16$ & $-6,78$ & $-6,44$ \\
51 & Regensburg & $-6,12$ & $-3,72$ & $-1,08$ & $-2,62$ \\
52 & Erlangen & $-1,22$ & $-1,70$ & $-0,36$ & $-0,48$ \\
53 & Fürth & $-1,26$ & $-2,30$ & 1,42 & 1,36 \\
54 & Nürnberg & $-2,72$ & $-1,54$ & $-1,74$ & $-2,18$ \\
55 & Würzburg & $-1,40$ & 2,36 & 2,24 & 0,74 \\
56 & Augsburg & $-2,94$ & $-0,38$ & $-1,12$ & 0,60 \\
57 & Berlin & $-3,98$ & $-1,80$ & $-1,02$ & $-0,52$ \\
58 & Potsdam & $-0,52$ & 0,20 & 4,98 & 7,24 \\
59 & Rostock & $-0,50$ & 2,18 & 0,26 & 1,98 \\
60 & Chemnitz & $-5,70$ & $-0,70$ & 0,40 & 0,82 \\
61 & Dresden & $-2,76$ & 2,30 & 2,02 & 1,24 \\
62 & Leipzig & $-10,72$ & 1,10 & 1,60 & 2,86 \\
63 & Halle & $-9,52$ & $-1,76$ & 0,92 & 1,70 \\
64 & Magdeburg & $-4,52$ & $-0,32$ & $-0,16$ & 1,02 \\
65 & Erfurt & $-0,56$ & 1,90 & 0,24 & 1,54 \\
66 & Jena & 0,38 & 3,74 & 2,04 & 0,90 \\
\hline & & & & &
\end{tabular}

nach der Wende in großer Zahl in den Westen gezogen waren, teils um Personen aus den ländlichen Gebieten Ostdeutschlands, in denen der Bevölkerungsrückgang zu erheblichen Infrastrukturdefiziten geführt hat. Ruhestandsmigranten waren schon immer zu einem erheblichen Teil Rückwanderer. In den oben genannten Untersuchungen zur counterurbanisation handelte es sich aber in der Regel um Rückwanderer, die nach dem Erwerbsleben zurück in die ländlichen Gegenden ihrer Kindheit und Jugend ziehen. Im Fall der ostdeutschen Städte sind die Rückwanderer aber vielleicht Reurbanisierer, die in die sanierten und in neuem Glanz erstrahlenden ostdeutschen Kernstädte zurückkehren. Dies zu klären, wäre eine Aufgabe künftiger Forschungen.

Dass Senioren in Ostdeutschland in erheblicher Zahl den Dörfern den Rücken kehren und in die Städte abwandern, ist inzwischen durch eine Studie des Berlin-Instituts für Bevölkerung und Entwicklung belegt (Slupina/Damm/Klingholz 2016). Dort wird nicht nur festgestellt, dass das Wanderungsvolumen der Generation 65+ geringfügig von 28 auf 30 Wanderungsbewegungen pro 1.000 Einwohner gestiegen ist, sondern dass es einen deutlichen Unterschied zwischen den auf dem Land und den in der Stadt wohnenden Senioren gibt: „Am stärksten war der Mobilitätsanstieg bei der älteren Dorfbevölkerung. Sie war bereits im Jahr 2008 am umzugsfreudigsten und hat diesen Vorsprung bei 2013 weiter ausgebaut. Denn in den größeren Städten zeigten sich im gleichen Zeitraum kaum Veränderungen beim Umzugsverhalten der Älteren. Hier scheint es weiterhin nur wenige 
Abbildung 6 Migrationssalden von 70 Großstädten im Vergleich 1995-1999 zu 20052009

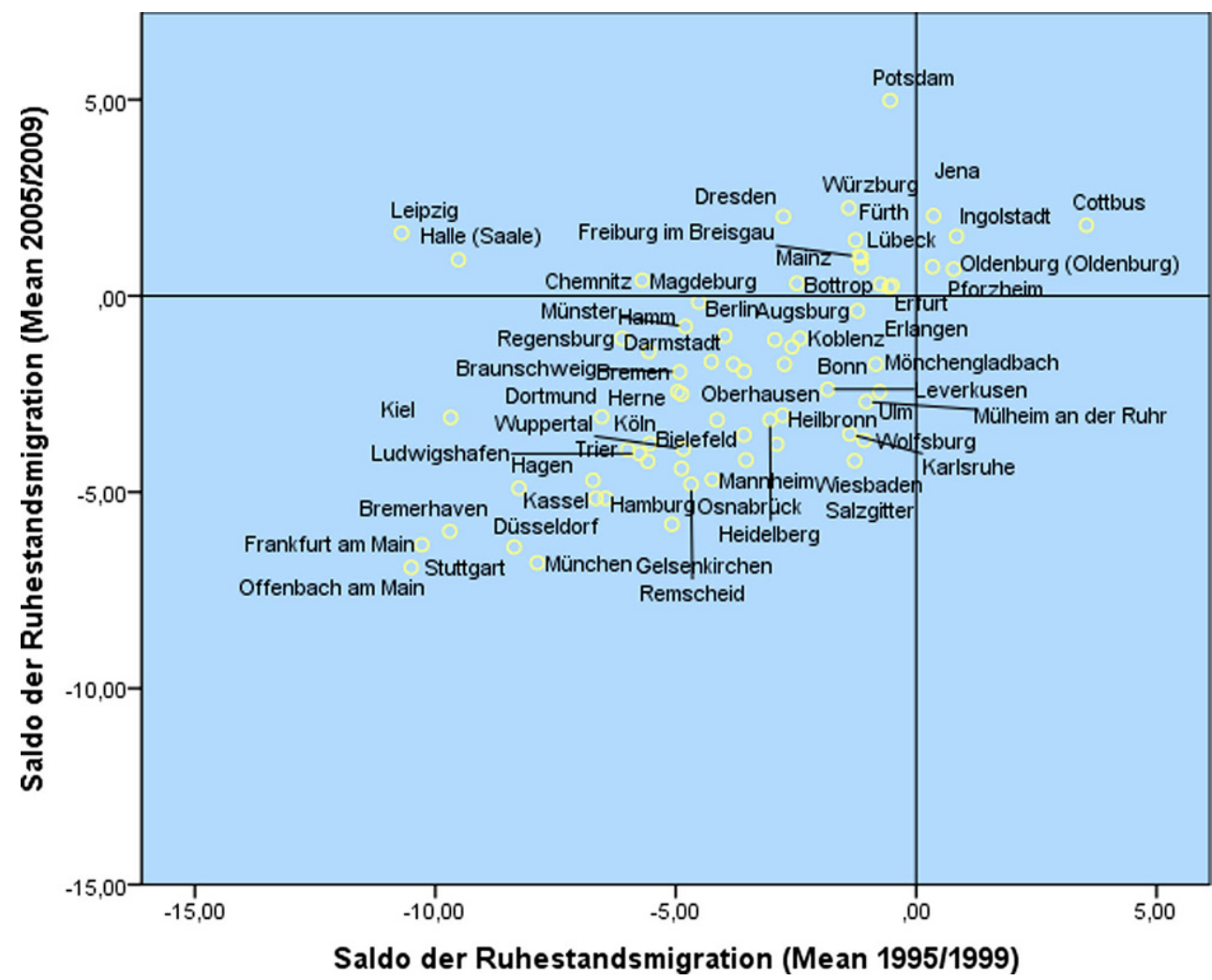

Saldo der Ruhestandsmigration (Mean 1995/1999)
Gründe für einen Wohnungswechsel im Alter zu geben“ (Slupina/Damm/Klingholz 2016: 43 f.). Je kleiner die Gemeinde, desto höher ist die Nettoabwanderung. Wachsende Infrastrukturdefizite im schrumpfenden ländlichen Raum in Verbindung mit der wachsenden Attraktivität der sanierten Städte führen hier zu klassischen (Re-)Urbanisierungsprozessen, die die Autoren als ,Wanderung in die Versorgungszentren“ (Slupina/Damm/Klingholz 2016: 46) charakterisieren. Allerdings wird in der Studie mit Nachdruck betont, dass es weniger die Großstädte sind (mit Ausnahme von Potsdam), die Ziel der Ruhestandsmigration werden, sondern eher die kleinen und mittleren Städte auch im peripheren Raum (Slupina/Damm/Klingholz 2016: 45).

Faktoren wie Rückwanderung und Landflucht beeinflussen in Westdeutschland vermutlich weniger die Ruhestandsmigration. Hier gibt es vor allem die schwache Form der Reurbanisierung, den Rückgang der Abwanderungsverluste der Kernstädte. In Verbindung mit dem von Friedrich (2008) aufgezeigten leichten Rückgang des Wanderungsvolumens insgesamt muss man wohl von einem deutlichen Trend zum Ageing in Place ausgehen. Brühl, Echter, Frölich von Bodelschwingh et al. (2005) fanden in Befragungen heraus, dass Ältere, die in Innenstadtbezirke zugezogen sind, mit dem neuen Wohnstandort sehr zufrieden sind. Das ist zwar ein erwartbares Ergebnis, aber es wird komplettiert durch andere Befragungen der nicht umgezogenen älteren Bevölkerung im urbanen Raum, in der ähnliche $\mathrm{Zu}$ - friedenheiten unter den Alteingesessenen festgestellt werden. Kramer und Pfaffenbach (2009) finden in ihrer Umfrage unter ,jungen Alten“, bei denen eine gewisse Mobilitätsbereitschaft durchaus $\mathrm{zu}$ erwarten wäre, ebenfalls die ausgeprägte Präferenz für die „Persistenz“, das heißt den Wunsch, dort alt $\mathrm{zu}$ werden, wo man gerade lebt (vgl. auch Kappler 2013). Studien, in deren Fokus der ländliche Raum stand, sind aus einem anderen Blickwinkel zu gleichen Resultaten gekommen. So kommt beispielsweise eine Untersuchung in den Ländern Schleswig-Holstein und Mecklenburg-Vorpommern (dsn/analytix 2007) zu dem Ergebnis, dass man im strukturschwachen Norden Deutschlands keine Hoffnung darin setzen sollte, durch Ruhestandswanderer aus den Städten größere wirtschaftliche Impulse $\mathrm{zu}$ erhalten. Insgesamt scheinen nicht nur in den ehemals heruntergekommenen ostdeutschen Städten, sondern auch in Westdeutschland die Bedingungen für das ,Altwerden in der Stadt' besser geworden zu sein. Vielerorts haben sich die Lebensbedingungen in den urbanen Zentren verbessert (und das nicht nur für die Senioren). $\mathrm{Zu}$ nennen sind die Maßnahmen zur Verringerung der Umweltbelastungen (Luftverschmutzung, Lärm), zum Schutz der schwächeren Verkehrsteilnehmer, zur Aufwertung der Grünflächen und des öffentlichen Raumes und zur Förderung der Barrierefreiheit. Auch die Weiterentwicklung der Einkaufs-, Versorgungs- und kulturellen Infrastrukturen ist hier zu nennen. Eine Reihe von Push-Faktoren der Ruhestandsmigrati- 
on dürften so an Wirksamkeit verloren haben. Unterstützend kommt der Ausbau der sozialstaatlichen Absicherung (vor allem) im Alter durch eine Pflegeversicherung hinzu, die es vielen ermöglicht, auch bei erheblichem Hilfebedarf in der eigenen Wohnung zurechtzukommen. Zu erwähnen sind auch die vielfältigen Ansätze (und Fördermittel) zum seniorengerechten Umbau der bestehenden Bausubstanz und für alten- oder generationengerechten Neubau. Auch im Hinblick auf die Einstellungen, Präferenzen und Werte der jetzt und in absehbarer Zukunft ins Seniorenalter nachrückenden Kohorten kann vermutet werden, dass diese besser gebildete, sozioökonomisch vergleichsweise gut abgesicherte und sehr multi-kulti-erfahrene Generation ,stadtaffiner" ist als frühere Alterskohorten.

Von Politik und Wissenschaft wird das Ageing in Place meist als wünschenswert erachtet. Der siebte Altenbericht der Bundesregierung konstatiert: „Auch in der fachpolitischen Debatte wird der Schluss gezogen, dass vor allem das, Wohnen bleiben in der eigenen Häuslichkeit‘ ermöglicht werden sollte. ,Alt werden zu Hause“ ist derzeit das dominierende Paradigma" (Deutscher Bundestag 2016: 228). Dabei, so wird weiter festgestellt, ist mit diesem Ageing in Place meist das Verbleiben in der eigenen Wohnung gemeint und nur selten das Verbleiben im Quartier. Damit werde diese Immobilitätspräferenz auch problematisch, denn es gibt unerwünschte Remanenzeffekte. Ältere belegen zu große Wohnungen und für junge Familien fehlt der Wohnraum. Zudem sind nach wie vor die meisten Wohnungen nicht altengerecht, insbesondere nicht barrierefrei. Vor diesem Hintergrund regt der siebte Altenbericht der Bundesregierung (unter der Überschrift „Mehr Mobilität ermöglichen“; Deutscher Bundestag 2016: 227) an, den Umzug im Alter wieder als politische Gestaltungsaufgabe zu begreifen. Die städtische Wohnpolitik steht damit vor der Aufgabe, mehr Wahlmöglichkeiten für das Leben im Alter (in der Stadt) zu schaffen, als derzeit verfügbar sind. Je nach individuellem Bedarf gehören dazu sowohl Hilfen zum Verbleib in der vertrauten Wohnung als auch geplante Umzüge im Quartier, die gewährleisten, dass man ohne Verlust des sozialen Netzwerks vor Ort in eine altengerechte Wohnung (und nicht ins Heim) gelangt. ${ }^{16}$

\section{Literatur}

Berry, B. J. L. (1976): The Counterurbanization Process: Urban America since 1970. In: Berry, B. J. L. (Hrsg.): Urbanization and Counterurbanization. Beverly Hills, 17-30. = Urban Affairs Annual Reviews 11 .

\footnotetext{
16 Der Siebte Altenbericht entwickelt dazu vor allem Vorschläge zur Gestaltung der nachbarschaftlichen Beziehungen und zum Sozialraummanagement (Deutscher Bundestag 2016: 258 ff.; vgl. auch die von Heinze (2016) in diesem Zusammenhang aufgeführten Anforderungen an eine kommunale Wohnpolitik).
}

Bonaguidi, A.; Abrami, V. T. (1992): The metropolitan aging transition and metropolitan redistribution of the elderly in Italy. In: Rogers, A. (Hrsg.): Elderly migration and population redistribution. A Comparative Study. London, 143-162.

Brake, K.; Herfert, G. (Hrsg.) (2012): Reurbanisierung. Materialität und Diskurs in Deutschland. Wiesbaden. doi: 10.1007/978-3-53194211-7

Brühl, H.; Echter, C.-P.; Frölich von Bodelschwingh, F.; Jekel, G. (2005): Wohnen in der Innenstadt - eine Renaissance? Berlin. = Difu-Beiträge zur Stadtforschung 41.

Cribier, F. (1980): A European Assessment of Aged Migration. In: Research on Aging 2, 2, 255-270. doi: 10.1177/016402758022013

Cribier, F.; Kych, A. (1993): A comparison of retirement migration from Paris and London. In: Environment and Planning A 25, 10, 1399-1420. doi: 10.1068/a251399

Deutscher Bundestag (2016): Siebter Bericht zur Lage der älteren Generation in der Bundesrepublik Deutschland. Berlin. = Drucksache $18 / 10210$.

dsn; analytix (2007): Altenwanderung und seniorengerechte Infrastruktur. Endbericht Teil A (im Auftrag des Innenministeriums des Landes Schleswig-Holstein). Kiel.

Eizenhöfer, R.; Link, A. (2006): Marktchancen spezieller Wohnsiedlungen für die Generation ,55plus“? Potenziale des amerikanischen Wohnmodells „Sun City“ und Alternativen für Deutschland. In: RaumPlanung 126/127, 136-140.

Fokkema, C. M. (1996): Residential moving behaviour of the elderly. An explanatory analysis for the Netherlands. Amsterdam.

Friedrich, K. (1994): Wohnortswechsel im Alter. Aktuelle Ergebnisse geographischer Mobilitätsforschung im vereinten Deutschland. In: Zeitschrift für Gerontologie 27, 410-418.

Friedrich, K. (1995): Altern in räumlicher Umwelt. Sozialräumliche Interaktionsmuster älterer Menschen in Deutschland und in den USA. Heidelberg. doi: 10.1007/978-3-642-72495-4

Friedrich, K. (2008): Binnenwanderungen älterer Menschen - Chancen für Regionen im demographischen Wandel? In: Informationen zur Raumentwicklung 3/4, 185-192.

Glasze, G.; Graze, P. (2007): Raus aus Suburbia, rein in die Stadt? Studie zur zukünftigen Wohnmobilität von Suburbaniten der Generation 50+. In: Raumforschung und Raumordnung 65, 5, 467473.

Golant, S. M. (1987): Residential Moves by Elderly Persons to U.S. Central Cities, Suburbs, and Rural Areas. In: Journal of Geronto$\operatorname{logy}$ 42, 5, 534-539. doi: 10.1093/geronj/42.5.534

Heinze, R. G. (2016): Wohnen und Wohnumfeld im Siebten Altenbericht. In: Informationsdienst Altersfragen 43, 6, 11-18.

Herfert, G.; Osterhage, F. (2012): Wohnen in der Stadt: Gibt es eine Trendwende zur Reurbanisierung? Ein quantitativ-analytischer Ansatz. In: Brake, K.; Herfert, G. (Hrsg.): Reurbanisierung. Materialität und Diskurs in Deutschland. Wiesbaden, 86-112. doi: 10.1007/978-3-531-94211-7_6

Kappler, M. (2013): Ruhestandsmigration der deutschen Nachkriegskohorte. Umzugsneigungen und Umzugspläne im Übergang zum Ruhestand aus individueller Perspektive. Karlsruhe. Dissertation, Karlsruher Institut für Technologie (KIT).

Kauffmann, A. (2015): Im Fokus: Bevölkerungsentwicklung der ostdeutschen Städte seit 1990 - Fiktion oder Wirklichkeit? In: Wirtschaft im Wandel 21, 1, 8-11.

Köppen, B.; Mai, R.; Schlömer, C. (2007): Reurbanisierung in Ostdeutschland - möglicher Leittrend zukünftiger Stadtentwicklung? In: Geographische Zeitschrift 95, 4, 211-230.

Kontuly, T.; Wiard, S.; Vogelsang, R. (1986): Counterurbanization in the Federal Republic of Germany. In: The Professional Geographer 38, 2, 170-181. doi: 10.1111/j.0033-0124.1986.00170.x

Kramer, C.; Pfaffenbach, C. (2009): Persistence preferred - on future residential (im)mobility among the generation 50plus. In: Erdkunde 63, 2, 161-172. doi: 10.3112/erdkunde.2009.02.04 
Kramer, C.; Pfaffenbach, C. (2011): Junge Alte als neue „Urbaniten“? Mobilitätstrends der Generation 50plus. In: Raumforschung und Raumordnung 69, 2, 79-90. doi: 10.1007/s13147-011-0078-2

Lewis, G.; McDermott, P.; Sherwood, K. B. (1991): The counterurbanization process: demographic restructuring and policy response in rural England. In: Sociologia Ruralis 31, 4, 309-320. doi: 10.1111/j.1467-9523.1991.tb00911.x

Litwak, E.; Longino, C. F. Jr. (1987): Migration Patterns among the Elderly: A Developmental Perspective. In: The Gerontologist 27, 3, 266-272. doi: 10.1093/geront/27.3.266

Longino, C. F. (1980): Residential Relocation of Older People. Metropolitan and Nonmetropolitan. In: Research on Aging 2, 2, 205216. doi: $10.1177 / 016402758022009$

Longino, C. F. (1995): Retirement Migration in America. Houston.

Milbert, A. (2017): Wie viel (Re-)Urbanisierung durchzieht das Land? Bonn. $=$ BBSR-Analysen Kompakt 07/2017.

Mitchell, C. J. A. (2004): Making sense of counterurbanization. In: Journal of Rural Studies 20, 1, 15-34. doi: 10.1016/S07430167(03)00031-7

Northcott, H. C.; Petruik, C.R. (2011): The Geographic Mobility of Elderly Canadians. In: Canadian Journal of Aging 30, 3, 311-322. doi: $10.1017 / \mathrm{S} 0714980811000262$

Nuissl, H.; Bigalke, B. (2007): Altwerden am Stadtrand - zum Zusammenhang von Suburbanisierung und demographischem Wandel. In: Raumforschung und Raumordnung 65, 5, 381-392.

Opaschowski, H. W. (2005): Besser leben, schöner wohnen? Leben in der Stadt der Zukunft. Darmstadt.

Osterhage, F.; Kaup, S. (2012): Reurbanisierung als neue Phase der Stadtentwicklung? Eine Analyse der Bevölkerungs- und Beschäftigtenentwicklung in Deutschland 1999 bis 2009. In: Pohlan, J.; Glasauer, H.; Hannemann, C.; Pott, A. (Hrsg.): Jahrbuch StadtRegion 2011/2012. Leverkusen-Opladen, 125-141.

Poddig, B. (2006): Die „Neuen Alten“im Wohnungsmarkt - Aktuelle Forschungsergebnisse über eine stark wachsende Zielgruppe. In: Forum Wohnen und Stadtentwicklung 3, 211-217.

Rees, P. (1992): Elderly migration and population redistribution in the United Kingdom. In: Rogers, A. (Hrsg.): Elderly migration and population redistribution. A Comparative Study. London, 203225.

Rohr-Zänker, R. (1989): A review of the literature on elderly migration in the Federal Republic of Germany. In: Progress in Human Geography 13, 2, 209-221. doi: 10.1177/030913258901300203

Rosenmayr, L. (1983): Die späte Freiheit. Das Alter, ein Stück bewußt gelebten Lebens. Berlin.

Schwarck, C. (2008): Wohin zieht es ältere Menschen in Baden-Württemberg. In: Statistisches Monatsheft Baden-Württemberg 5, 1418.

Serow, W. J. (1987): Why the Elderly Move: Cross-national Comparisons. In: Research on Aging 9, 4, 582-597. doi: 10.1177/ 0164027587094006

Slupina, M.; Damm, T.; Klingholz, R. (2016): Im Osten auf Wanderschaft. Wie Umzüge die demografische Landkarte zwischen Rügen und Erzgebirge verändern. Berlin.

Spars, G. (2012): Neue Attraktivität der Städte - Herausforderungen für die kommunale Politik? In: Forum Wohnen und Stadtentwicklung 1, 47-52.

Statistisches Bundesamt (2015): Zeitverwendungserhebung. Aktivitäten in Stunden und Minuten für ausgewählte Personengruppen. Wiesbaden.

Stockdale, A.; Findlay, A.; Short, D. (2000): The repopulation of rural Scotland: opportunity and threat. In: Journal of Rural Studies 16, 2, 243-257. doi: 10.1016/S0743-0167(99)00045-5

Walters, W. H. (1994): Place characteristics in elderly migration research. In: Bulletin of Bibliography 51, 4, 341-354.

Walther, U.-J. (2007): Alternde Stadtbevölkerung - Altern in der Stadt. In: Baum, D. (Hrsg.): Die Stadt in der sozialen Arbeit. Ein Handbuch für soziale und planende Berufe. Wiesbaden, 276-286.

Warnes, A. M.; Law, C. M. (1983): The elderly population of Great Britain: locational trends and policy implications. In: Transactions of the Institute of British Geographers 9, 1, 37-59.

Wiseman, R. F. (1980): Why Older People Move: Theoretical Issues. In: Research on Aging 2, 2, 141-154. doi: 10.1177/ 016402758022003 